\title{
On the Degree Sequences of Uniform Hypergraphs
}

\author{
Andrea Frosini, Christophe Picouleau, and Simone Rinaldi \\ Dipartimento di Sistemi e Informatica, Università di Firenze, \\ Viale Morgagni, 65 - 50134 Firenze - Italy \\ Laboratoire CEDRIC, CNAM \\ 292 rue St Martin, 75003 Paris - France \\ Dipartimento di Matematica e Informatica, Università di Siena, \\ Pian dei Mantellini, 44 - 53100 Siena - Italy \\ andrea.frosini@unifi.it, christophe.picouleau@cnam.fr, rinaldi@unisi.it
}

\begin{abstract}
In hypergraph theory, determining a good characterization of $d$, the degree sequence of an $h$-uniform hypergraph $\mathcal{H}$, and deciding the complexity status of the reconstruction of $\mathcal{H}$ from $d$, are two challenging open problems. They can be formulated in the context of discrete tomography: asks whether there is a matrix $A$ with nonnegative projection vectors $H=(h, h, \ldots, h)$ and $V=\left(d_{1}, d_{2}, \ldots, d_{n}\right)$ with distinct rows.

In this paper we consider the subcase where the vectors $H$ and $V$ are both homogeneous vectors, and we solve the related consistency and reconstruction problems in polynomial time. To reach our goal, we use the concepts of Lyndon words and necklaces of fixed density, and we apply some already known algorithms for their efficient generation.
\end{abstract}

Keywords: Discrete Tomography, Reconstruction problem, Lyndon word, Necklace, hypergraph degree sequence.

\section{Introduction}

The degree sequence, also called graphic sequence, of a simple graph (a graph without loop or parallel edges) is the list of vertex degrees, usually written in nonincreasing order, as $d=\left(d_{1}, d_{2}, \ldots, d_{n}\right), d_{1} \geq d_{2} \geq \cdots \geq d_{n}$. The problem of characterizing the graphic sequences of graphs was solved by Erdös and Gallai $($ see [4]):

Theorem 1. (Erdös, Gallai) A sequence $d=\left(d_{1}, d_{2}, \ldots, d_{n}\right)$ where $d_{1} \geq d_{2} \geq$ $\cdots \geq d_{n}$ is graphic if and only if $\sum_{i=1}^{n} d_{i}$ is even and

$$
\sum_{i=1}^{k} d_{i} \leq k(k-1)+\sum_{i=k+1}^{n} \min \left\{k, d_{i}\right\}, 1 \leq k \leq n .
$$

An hypergraph $\mathcal{H}=(V, \mathcal{E})$ is defined as follows (see [5]): $\operatorname{Vert}=\left\{v_{1}, \ldots, v_{n}\right\}$ is a ground set of vertices and $\mathcal{E} \subset 2^{|V e r t|} \backslash \emptyset$ is the set of hyperedges such that $e \not \subset e^{\prime}$ for any pair $e, e^{\prime}$ of $\mathcal{E}$. The degree of a vertex $v \in V e r t$ is the number of hyperedges $e \in \mathcal{E}$ such that $v \in e$. An hypergraph $\mathcal{H}=(V e r t, \mathcal{E})$ is $h$-uniform

R. Gonzalez-Diaz, M.-J. Jimenez, B. Medrano (Eds.): DGCI 2013, LNCS 7749, pp. 300-310, 2013.

(C) Springer-Verlag Berlin Heidelberg 2013 
if $|e|=h$ for all hyperedge $e \in \mathcal{E}$. Moreover $\mathcal{H}=($ Vert, $\mathcal{E})$ has no parallel hyperedges,i.e., $e \neq e^{\prime}$ for any pair $e, e^{\prime}$ of hyperedges. Thus a simple graph (loopless and without parallel edges) is a 2-uniform hypergraph.

The problem of the characterization of the degree sequences of $h$-uniform hypergraphs is one of the most relevant among the unsolved problems in the theory of hypergraphs [5] even for the case of 3-uniform hypergraphs. Also its complexity status is still open.

This problem has been related to a class of problems that are of great relevance in the field of discrete tomography. More precisely the aim of discrete tomography is the retrieval of geometrical information about a physical structure, regarded as a finite set of points in the integer lattice, from measurements, generically known as projections, of the number of atoms in the structure that lie on lines with fixed scopes. A common simplification is to represent a finite physical structure as a binary matrix, where an entry is 1 or 0 according to the presence or absence of an atom in the structure at the corresponding point of the lattice. One of the challenging problems in the field is then to reconstruct the structure, or, at least, to detect some of its geometrical properties from a small number of projections. One can refer to the books of G.T. Herman and A. Kuba 14 15 for further information on the theory, algorithms and applications of this classical problem in discrete tomography.

Here we recall the seminal result in the field of the discrete tomography due to Ryser [19. Let $H=\left(h_{1}, \ldots, h_{m}\right), h_{1} \geq h_{2} \geq \cdots \geq h_{m}$, and $V=$ $\left(v_{1}, \ldots, v_{n}\right), v_{1} \geq v_{2} \geq \cdots \geq v_{n}$, be two nonnegative integral vectors, and $\mathcal{U}(H, V)$ be the class of binary matrices $A=\left(a_{i j}\right)$ satisfying

$$
\begin{gathered}
\sum_{j=1}^{n} a_{i j}=h_{i} 1 \leq i \leq m \\
\sum_{i=1}^{m} a_{i j}=v_{j} 1 \leq j \leq n
\end{gathered}
$$

In this context $H$ and $V$ are called the row, respectively column, projection of $A$, as depicted in Fig. 11 Denoting by $\bar{V}=\left(\overline{v_{1}}, \overline{v_{2}}, \ldots\right)$ the conjugate sequence, also called the Ferrer sequence, of $V$ where $\bar{v}_{i}=\left|\left\{v_{j}: v_{j} \in V, v_{j} \geq i\right\}\right|$. Ryser gave the following [19]:

Theorem 2. (Ryser) $\mathcal{U}(H, V)$ is nonempty if and only if

$$
\begin{aligned}
& \sum_{i=1}^{m} h_{i}=\sum_{i=1}^{n} v_{i} \\
& \sum_{j=1}^{i} h_{j} \geq \sum_{j=1}^{i} \bar{v}_{j} \quad \forall i \in\{1, \ldots, m\}
\end{aligned}
$$

Moreover this characterization, and the reconstruction of $A$ from its two projections $H$ and $V$, can be done in polynomial time (see [14]). Some applications in discrete tomography requiring additional constraints can be found in 17]2 11/16 17 18 23 .

As shown in 4 this problem is equivalent to the reconstruction of a bipartite graph $G=(H, V, E)$ from its degree sequences $H=\left(h_{1}, \ldots, h_{m}\right)$ and $V=$ $\left(v_{1}, \ldots, v_{n}\right)$. Numerous papers give some generalizations of this problem for the graphs with colored edges (see [36 91013]). 
So, in this context, the problem of the characterization of the degree sequence $\left(d_{1}, d_{2}, \ldots, d_{n}\right)$ of an $h$-uniform hypergraph $\mathcal{H}$ (without parallel edges) asks whether there is a binary matrix $A \in \mathcal{U}(H, V)$ with nonnegative projection vectors $H=(h, h, \ldots, h)$ and $V=\left(d_{1}, d_{2}, \ldots, d_{n}\right)$ with distinct rows, i.e., $A$ is the incidence matrix of $\mathcal{H}$ where rows and columns correspond to hyperedges and vertices, respectively. To our knowledge the problem of the reconstruction of a binary matrix with distinct rows has not be studied in discrete tomography.

In this paper, we carry on our analysis in the special case where the $h$-uniform hypergraph to reconstruct is also $d$-regular, i.e., each vertex $v$ has the same degree $d$, in other words the vector of the vertical projection is homogeneous, i.e. $V=(d, \ldots, d)$.

The studies in this paper focuses both on the decision problem, and on the related reconstruction problem, i.e. the problem of determining an element of $\mathcal{U}(H, V)$ consistent with $H$ and $V$. To accomplish this task we will design an algorithm that runs in polynomial time with respect to $m$ and $n$ the dimensions of the matrix to reconstruct. This algorithm uses the concepts of Lyndon words and necklaces of fixed density, also we apply some already known algorithms for their efficient generation.

\section{Definitions and Introduction of the Problems}

Let $A$ be a binary matrix having $m$ rows and $n$ columns, and let us consider the two integer vectors $H=\left(h_{1}, \ldots, h_{m}\right)$ and $V=\left(v_{1}, \ldots, v_{n}\right)$ of its horizontal and vertical projections, respectively, as defined in Section 1 (see Fig. 1).

$\begin{array}{cllllllll}V= & 3 & 1 & 2 & 2 & 6 & 3 & 2 & 4 \\ 3 & 0 & 0 & 1 & 0 & 1 & 0 & 0 & 1 \\ 2 & 1 & 0 & 0 & 0 & 0 & 1 & 0 & 0 \\ 0 & 0 & 0 & 0 & 0 & 0 & 0 & 0 & 0 \\ 0 & 0 & 0 & 0 & 0 & 0 & 0 & 0 & 0 \\ 2 & 1 & 0 & 0 & 0 & 0 & 0 & 0 & 1 \\ 7 & 0 & 1 & 1 & 1 & 1 & 1 & 1 & 1 \\ 1 & 0 & 0 & 0 & 0 & 1 & 0 & 0 & 0 \\ 2 & 0 & 0 & 0 & 0 & 1 & 0 & 1 & 0 \\ 3 & 0 & 0 & 0 & 0 & 1 & 1 & 0 & 1 \\ 3 & 1 & 0 & 0 & 1 & 1 & 0 & 0 & 0\end{array}$

Fig. 1. A binary matrix, used in discrete tomography to represent finite discrete sets, and its vectors $H$ and $V$ of horizontal and vertical projections, respectively

These few notions are enough to state the general version of the problems we will consider in this paper (in fact, we consider more specialized versions, as defined further below): 


\section{Consistency $(H, V, \mathcal{C})$}

Input: two integer vectors $H$ and $V$, and a class of discrete sets $\mathcal{C}$.

Question: does there exist an element of $\mathcal{C}$ whose horizontal and vertical projections are $H$ and $V$, respectively?

\section{Reconstruction $(H, V, \mathcal{C})$}

Input: two integer vectors $H$ and $V$, and a class of discrete sets.

Task: reconstruct a matrix $A \in \mathcal{C}$ whose horizontal and vertical projections are $H$ and $V$, respectively, if it exists, otherwise give failure.

In the sequel we are going to consider the class of binary matrices having no equal rows and homogeneous horizontal projections, denoted $\mathcal{E}$, due to its connections, as mentioned in the Introduction, with the characterization of the degree sequences of $h$-uniform hypergraphs.

In 20, Ryser gave a characterization of the instances of Consistency $(H, V, \mathcal{C})$, with $\mathcal{C}$ being the class of the binary matrices, that admit a positive answer, after noticing that the following conditions are necessary for the existence of a matrix consistent with two generic vectors $H$ and $V$ of projections:

Condition 1: for each $1 \leq i \leq m$ and $1 \leq j \leq n$, it holds $h_{i} \leq n$ and $v_{j} \leq m$; Condition 2: $\sum_{i=1}^{m} h_{i}=\sum_{j=1}^{n} v_{j}$,

and then he added a further condition in order to obtain the characterization of the instances that admit a solution, as recalled in the Introduction.

The authors of [8], pointed out that these two conditions turn out to be sufficient in case of homogeneous horizontal and vertical projections, by showing their maximality w.r.t. the cardinality of the related sets of solutions.

Ryser defined a well known greedy algorithm to solve Reconstruction $(H, V, \mathcal{C})$ that does not compare the obtained rows, and does not admit an easy generalization to perform this further task.

So, since we want to restrict ourself to deal with matrices having different rows, and homogeneous horizontal projections, we approach the two problems in a different way, considering each row as a binary word, and grouping them into equivalence classes according to their cyclic shifts, as defined in the next section. Now, we state a third necessary condition for answering to $\operatorname{Consistency}(H, V, \mathcal{E})$ :

Condition 3: Consistency $(H, V, \mathcal{E})$ has a negative answer if the dimension of the vector $H$ is greater than $\left(\begin{array}{l}n \\ h\end{array}\right)$.

In other words, there does not exist a matrix having $H$ and $V$ as homogeneous projections, and more than $\left(\begin{array}{l}n \\ h\end{array}\right)$ different rows; this result can be easily deduced using a cardinality argument. From our analysis, we will prove that the three conditions are also sufficient to solve in linear time the problem $\operatorname{Consistency}(H, V, \mathcal{E})$ with homogeneous $H$ and $V$. 


\section{The Problem Consistency $(H, V, \mathcal{E})$}

Let us face the consistency problem for the class $\mathcal{E}$ on the homogeneous instance $H$ and $V$ from a different perspective, by observing that each row of a binary solution matrix can be regarded as a binary finite word $u=u_{1} u_{2} \ldots u_{n}$, whose length $n$ is the number of columns of the matrix, and whose number $h$ of 1elements is the common horizontal projection.

We note that applying a cyclic shift to the word $u$, denoted by $s(u)$, we obtain a different word $s(u)=u_{2} u_{3} \ldots u_{n} u_{1}$, unless the cases $u=(1)^{n}$ or $u=(0)^{n}$, of the same length, and having the same number of elements 1 inside, so it can be considered as a possible row different from $u$ of a solution matrix. Iterating the shift of a word $u$, one can think to easily obtain a sequence of words that row wise arranged inside a matrix, lead to a solution of $\operatorname{Consistency}(H, V, \mathcal{E})$. We indicate with $s^{k}(u)$, where $k \geq 0$, the application of $k$ times the shift operator to the word $u$. Unfortunately, two problems may occur: the words repeat after at most $n$ shifts, and the vertical projections may not reach the desired value $v$, i.e. the arranged shifts form a submatrix of a solution matrix (see Fig. 2). The following trivial property holds:

Property 1. Let $u$ be a binary word of length $n$ having $h \leq n$ 1-elements inside. Let us consider the $n \times n$ matrix $A$ obtained by row wise arranging all the cyclic shifts of $u$. Then, $A$ has all horizontal and vertical projections equal to $h$.

One can easily notice that the rows of the matrix $A$ may not all be different. Throughout the paper we will denote by $M(u)$ the matrix obtained by row wise arranging all the different cyclic shifts of a word $u$. To establish how many different rows can be obtained by shifting a given binary word, we need to recall the definitions and main properties of necklaces and Lyndon words.

Following the notation in [21, a binary necklace (briefly necklace) is an equivalence class of binary words under cyclic shift. We identify a necklace with the lexicographically least representative $u$ in its equivalence class, denoted by $[u]$.

The set of all (the words representative of) the necklaces with length $n$ is denoted $N(n)$. For example,

$$
N(4)=\{0000,0001,0011,0101,0111,1111\} \text {. }
$$

An important class of necklaces are those that are aperiodic. An aperiodic (i.e. period $\geq n$ ) necklace is called a Lyndon word. Let $L(n)$ denote the set of all Lyndon words with length $n$. For example, $L(4)=\{0001,0011,0111\}$.

We denote fixed-density necklaces, Lyndon words in a similar manner by adding the additional parameter $d$ to represent the number of $1 \mathrm{~s}$ in the words. We refer to the number $d$ as the density of the word. Thus the set of necklaces with density $d$ is represented by $N(n, d)$, and the set of Lyndon words with density $d$ is represented by $L(n, d)$. For example, $N(4,2)=\{0011,0101\}$, and $L(4,2)=\{0011\}$. 
It is known from Gilbert and Riordan 12 that the number of fixed density necklaces and Lyndon words is

$$
N(n, d)=\frac{1}{n} \sum_{j \backslash \operatorname{gcd}(n, d)} \phi(j)\left(\begin{array}{l}
n / j \\
d / j
\end{array}\right), \quad L(n, d)=\frac{1}{n} \sum_{j \backslash \operatorname{gcd}(n, d)} \mu(j)\left(\begin{array}{l}
n / j \\
d / j
\end{array}\right)
$$

respectively, where the symbols $\phi$ and $\mu$ refer to the Euler and Möbius functions. Now we enlighten the connection between these objects and our problem, refining Property 1.

Proposition 1. If $u$ is a word of length $n$ and density $h \leq n$, then the cardinality of $[u]$ (i.e. the number of rows of $M(u)$ ) is a divisor of $n$.

As a consequence, we have the following refinements:

Proposition 2. If $u$ is a Lyndon word of length $n$ and density $h$, then the cardinality of $[u]$ (i.e. the number of rows of $M(u)$ ) is equal to $n$, and the vertical projections of $M(u)$ are all equal to $h$.

Figure 2 shows the 12 different cyclic shifts of the Lyndon word $u=(0)^{6}(1)^{6}$ arranged in the first 12 rows of the matrix. All the rows of $M(u)$ have horizontal and vertical projections equal to the density of $u$, i.e. 6 .

$\left.\begin{array}{|llllllllllll|}\hline 0 & 0 & 0 & 0 & 0 & 0 & 1 & 1 & 1 & 1 & 1 & 1 \\ \hline 1 & 0 & 0 & 0 & 0 & 0 & 0 & 1 & 1 & 1 & 1 & 1 \\ 1 & 1 & 0 & 0 & 0 & 0 & 0 & 0 & 1 & 1 & 1 & 1 \\ 1 & 1 & 1 & 0 & 0 & 0 & 0 & 0 & 0 & 1 & 1 & 1 \\ 1 & 1 & 1 & 1 & 0 & 0 & 0 & 0 & 0 & 0 & 1 & 1 \\ 1 & 1 & 1 & 1 & 1 & 0 & 0 & 0 & 0 & 0 & 0 & 1 \\ 1 & 1 & 1 & 1 & 1 & 1 & 0 & 0 & 0 & 0 & 0 & 0 \\ 0 & 1 & 1 & 1 & 1 & 1 & 1 & 0 & 0 & 0 & 0 & 0 \\ 0 & 0 & 1 & 1 & 1 & 1 & 1 & 1 & 0 & 0 & 0 & 0 \\ 0 & 0 & 0 & 1 & 1 & 1 & 1 & 1 & 1 & 0 & 0 & 0 \\ 0 & 0 & 0 & 0 & 1 & 1 & 1 & 1 & 1 & 1 & 0 & 0 \\ 0 & 0 & 0 & 0 & 0 & 1 & 1 & 1 & 1 & 1 & 1 & 0 \\ 0 & 0 & 1 & 1 & 0 & 0 & 1 & 1 & 0 & 0 & 1 & 1 \\ 1 & 0 & 0 & 1 & 1 & 0 & 0 & 1 & 1 & 0 & 0 & 1 \\ 1 & 1 & 0 & 0 & 1 & 1 & 0 & 0 & 1 & 1 & 0 & 0 \\ 0 & 1 & 1 & 0 & 0 & 1 & 1 & 0 & 0 & 1 & 1 & 0 \\ 0 & 1 & 0 & 1 & 0 & 1 & 0 & 1 & 0 & 1 & 0 & 1 \\ 1 & 0 & 1 & 0 & 1 & 0 & 1 & 0 & 1 & 0 & 1 & 0\end{array}\right]-M(000000111111)$

Fig. 2. A solution to Reconstruction $(H, V, \mathcal{C})$ when the horizontal projections have constant value 6 , and the vertical projections 9 . The submatrices $M(u)$ obtained by row wise arranging the elements of three necklaces are highlighted. Note that $M\left((0011)^{3}\right)$ and $M\left((01)^{6}\right)$ are the only two possible necklaces of length 12 and density 6 having 4 and 2 rows, respectively. 
Proposition 3. If $u=v^{k}$ (i.e. $u=v \ldots v, k$ times), with $k=\operatorname{gcd}\{n, h\}$, is a necklace of length $n$ and density $h$, and $v$ a Lyndon word, then the cardinality of $[u]$ is equal to $n / k$, and the vertical projections of $M(u)$ are all equal to $h / k$.

Figure 2 shows the $12 / 3=4$ different cyclic shifts of the word $u=(0011)^{3}$ arranged from row 13 to row 16 of the matrix, and the $12 / 6=2$ different cyclic shifts of the word $v=(01)^{6}$ in rows 17 and 18. All the rows of $\mathrm{M}(\mathrm{u})$ have horizontal projections equal to 6 and vertical projections equal to $6 / 3=2$, while the rows of $M(v)$ have horizontal projections equal to 6 and vertical projections equal to $6 / 6=1$.

In the following we will prove that a pair $H$ and $V$ of projections satisfy Conditions 1,2 , and 3 if and only if they are consistent with a matrix in $\mathcal{E}$, solving Consistency $(H, V, \mathcal{E})$.

Let $d_{0}=1, d_{1}, d_{2}, \ldots, d_{t}$ be the increasing sequence of the common divisors of $n$ and $h$. The following equation holds:

$$
\left(\begin{array}{l}
n \\
h
\end{array}\right)=\sum_{i=0 \ldots, t} \frac{n}{d_{i}} L\left(\frac{n}{d_{i}}, \frac{h}{d_{i}}\right) .
$$

This equation is an immediate consequence of the fact that each word of length $n$ and density $h$ belongs to exactly one necklace.

Theorem 3. Let $H$ and $V$ be two homogeneous vectors of projections of dimension $m$ and $n$, and elements $h$ and $v$, respectively, satisfying Conditions 1, 2, and 3 , i.e. being a valid instance of Consistency $(H, V, \mathcal{E})$. Then, there exists a Lyndon word $L\left(n / d_{i}, h / d_{i}\right)$ such that $n / d_{i} \leq m$.

Proof. Let us proceed by contradiction assuming that there does not exist a Lyndon word whose length is $n / d<m$, for each $d \in d_{1}, \ldots, d_{t}$. Since $H$ and $V$ are homogeneous, and satisfy Conditions 1 and 2, then there exists a matrix $A$ having $H$ and $V$ as projections (a consequence of Ryser's characterization of solvable instances, as stated in 8, Theorem 3).

Let us assume that $d=d_{t}=\operatorname{gcd}\{n, h\}, h^{\prime}=h / d$, and $n^{\prime}=n / d$; by Condition 1 , it holds

$$
h^{\prime} m=v n^{\prime}
$$

with $n^{\prime}$ and $h^{\prime}$ coprime, so $v=h^{\prime}\left(m / n^{\prime}\right)$, and $n^{\prime}$ divides $m$. The hypothesis $n^{\prime}>m$ leads to a contradiction.

Theorem 3 can be rephrased saying that if $H$ and $V$ are homogeneous consistent vectors of projections, then there exists a solution that contains all the elements of a necklace $[u]$. The solution in linear time of Consistency $(H, V, \mathcal{E})$ is a neat consequence:

Corollary 1. Let $H$ and $V$ be two homogeneous vectors satisfying Conditions 1,2 , and 3. There always exists a matrix having different rows, and $H$ and $V$ as projections. 
The result of Theorem 3, together with the following proposition that point out a property of the necklace whose representant is $u=(0)^{n-h}(1)^{h}$, will be used in the next section to solve Reconstruction $(H, V, \mathcal{E})$.

Proposition 4. Let $u^{\prime}$ be an element of the class $[u]$, with $u=(0)^{n-h}(1)^{h}$. The elements $u^{\prime}, s^{h}\left(u^{\prime}\right), s^{2 h}\left(u^{\prime}\right), \ldots, s^{(k-1) h}\left(u^{\prime}\right)$, with $k=n / \operatorname{gcd}\{n, h\}$, forms $a$ subclass of $[u]$, and they can be arranged in a matrix $A^{\prime}$ such that

1. the vertical projections of $A^{\prime}$ are homogeneous and equal to $h / \operatorname{gcd}\{n, h\}$;

2. $A^{\prime}$ is minimal with respect to the number of rows among the matrices having $H$ as horizontal projections, and homogeneous vertical projections.

The proof directly follows from the properties of the greatest common divisor. Let us denote with $M(u)_{1}, \ldots, M(u)_{\operatorname{gcd}\{n, h\}}$ the matrices defined in Proposition 4 .

\section{An Algorithm to Solve Reconstruction $(H, V, \mathcal{E})$}

We start recalling that in 22 a constant amortized time (CAT) algorithm FastFixedContent for the exhaustive generation of necklaces of fixed length and density $N(n, h)$ is presented. The author then shows that a slight modification of his algorithm can also be applied for the CAT generation of Lyndon words $L(n, h)$ of fixed length and density. In particular, his algorithm -here denoted GenLyndon $(n, h)$ - constructs a generating tree of the words, and since the tree has height $h$, the computational cost of generating $k$ words of $L(n, h)$ is $O(k \cdot h \cdot n)$.

Our reconstruction algorithm works as follows:

$\boldsymbol{\operatorname { R e c }}(H, V, \mathcal{E})$

Input : Two homogeneous vectors: $H=(h, \ldots, h)$ of length $m$, and $V=$ $(v, \ldots, v)$ of length $n$, satisfying Conditions 1,2 , and 3 .

Output : An element of the class $\mathcal{E}$ having $H$ and $V$ as horizontal and vertical projections, respectively.

Step 1: Let compute the sequence $d_{0}=1<d_{1}<d_{2}<\cdots<d_{t}$ of the common divisors of $n$ and $h$, and initialize the matrix $A_{-1}=\emptyset$.

Step 2: For $i=0$ to $t$ do:

Step 2.1: By applying $\operatorname{GenLyndon}(n, h)$, generate the sequence of $q=$ $\min \{\lfloor v / h\rfloor, L(n, h)\}$ Lyndon words, denoted $u_{1}, \ldots, u_{q}$. If $q \neq L(n, h)$, then do not include in the sequence the Lyndon word $(0)^{n-h}(1)^{h}$.

Step 2.2: Create the matrix $A_{i}$, obtained by row wise arranging the matrices $M\left(\left(u_{j}\right)^{d_{i}}\right)$, for $j=1, \ldots, q$.

Update $v=v-q \cdot h$.

If $v=0$ then output $A_{i}$,

else if $q \neq L(n, h)$, create the matrix $A$ obtained by row wise arranging the matrix $A_{i}$ with the column wise arranging of $d_{i}$ times the matrices $M(u)_{j}$, with $u=(0)^{n-h}(1)^{h}, j=1, \ldots, q^{\prime}$, and $q^{\prime}=v \cdot \operatorname{gcd}\{n, h\} / h$, else update $n=n / d_{i+1}$, and $h=h / d_{i+1}$. 
To better understand the reconstruction algorithm, we give a first simple example with the instance $H=(2, \ldots, 2)$ of length $m=15$, and $V=(5, \ldots, 5)$ of length $n=6$. In Step 1 the values $d_{0}=1$, and $d_{1}=2$ are set.

In Step 2, GenLyndon(6,2) generates $q=2$ Lyndon words, i.e. the words 000011 , and 000101 ; since $L(6,2)=2$, then the word 000011 is included in the sequence. Now the matrix $A_{0}$, depicted in Fig. 3] on the left, is created. Finally, the values $v=5-2 \cdot 2=1, n=6 / 2=3$, and $h=2 / 2=1$ are updated.

The second run of Step 2 starts, and GenLyndon(3,1) generates the Lyndon word 001. The matrix $A_{1}$ is created by row wise arranging $A_{0}$ with the matrix $M\left((001)^{2}\right)$ as shown in Fig. 3, on the right.

\begin{tabular}{lllllll|}
\hline 0 & 0 & 0 & 0 & 1 & 1 \\
\hline 1 & 0 & 0 & 0 & 0 & 1 \\
1 & 1 & 0 & 0 & 0 & 0 \\
0 & 1 & 1 & 0 & 0 & 0 \\
0 & 0 & 1 & 1 & 0 & 0 \\
0 & 0 & 0 & 1 & 1 & 0 \\
\hline 0 & 0 & 0 & 1 & 0 & 1 \\
\hline 1 & 0 & 0 & 0 & 1 & 0 \\
0 & 1 & 0 & 0 & 0 & 1 \\
1 & 0 & 1 & 0 & 0 & 0 \\
0 & 1 & 0 & 1 & 0 & 0 \\
0 & 0 & 1 & 0 & 1 & 0
\end{tabular}

\begin{tabular}{|llllll|}
\hline 0 & 0 & 0 & 0 & 1 & 1 \\
\hline 1 & 0 & 0 & 0 & 0 & 1 \\
1 & 1 & 0 & 0 & 0 & 0 \\
0 & 1 & 1 & 0 & 0 & 0 \\
0 & 0 & 1 & 1 & 0 & 0 \\
0 & 0 & 0 & 1 & 1 & 0 \\
\hline 0 & 0 & 0 & 1 & 0 & 1 \\
\hline 1 & 0 & 0 & 0 & 1 & 0 \\
0 & 1 & 0 & 0 & 0 & 1 \\
1 & 0 & 1 & 0 & 0 & 0 \\
0 & 1 & 0 & 1 & 0 & 0 \\
0 & 0 & 1 & 0 & 1 & 0 \\
0 & 0 & 1 & 0 & 0 & 1 \\
1 & 0 & 0 & 1 & 0 & 0 \\
0 & 1 & 0 & 0 & 1 & 0
\end{tabular}

Fig. 3. The solution of dimension $15 \times 6$ obtained by applying $\operatorname{Rec}(H, V, \mathcal{E})$ when the horizontal projections have constant value 2 , and the vertical projections 5

A second example concerns the use of the word $(0)^{n-h}(1)^{h}$ that in certain cases is set aside from the sequence of Lyndon words generated in Step 2: the instance we consider is $H=(3, \ldots, 3)$ of length $m=15$, and $V=(5, \ldots, 5)$ of length $n=9$. In Step 1 the values $d_{0}=1$, and $d_{1}=3$ are set.

In Step 2, GenLyndon $(9,3)$ generates $q=\min \{\lfloor 5 / 3\rfloor, L(9,3)\}=1$ Lyndon words, i.e. the word 000001011 ; since $q \neq L(9,3)$, then the word 000000111 is not included in the sequence. Now the matrix $A_{0}$, depicted in Fig. 4, on the left, is created. The value $v=5-3 \cdot 1=2$ is set.

Now, since $q \neq L(9,3), q^{\prime}=2(=2 \cdot \operatorname{gcd}\{9,3\} / 3)$ submatrices of $M(000000111)$ are computed, as defined in Proposition 4, and row wise arranged with $A_{0}$, obtaining the matrix in Fig. 4, on the right.

Note that without the use of the Lyndon word 000000111, the procedure is not able to reach the solution since in the second run of Step 2, $\operatorname{GenLyndon}(3,1)$ generates only one Lyndon word, i.e. 001, whose matrix $M\left((001)^{3}\right)$ has homogeneous vertical projections equal to 1 , not enough to reach the desired value 2 . 


\begin{tabular}{|lllllllll|}
\hline 0 & 0 & 0 & 0 & 0 & 1 & 0 & 1 & 1 \\
\hline 1 & 0 & 0 & 0 & 0 & 0 & 1 & 0 & 1 \\
1 & 1 & 0 & 0 & 0 & 0 & 0 & 1 & 0 \\
0 & 1 & 1 & 0 & 0 & 0 & 0 & 0 & 1 \\
1 & 0 & 1 & 1 & 0 & 0 & 0 & 0 & 0 \\
0 & 1 & 0 & 1 & 1 & 0 & 0 & 0 & 0 \\
0 & 0 & 1 & 0 & 1 & 1 & 0 & 0 & 0 \\
0 & 0 & 0 & 1 & 0 & 1 & 1 & 0 & 0 \\
0 & 0 & 0 & 0 & 1 & 0 & 1 & 1 & 0 \\
\end{tabular}

\begin{tabular}{|lllllllll|}
\hline 0 & 0 & 0 & 0 & 0 & 1 & 0 & 1 & 1 \\
\hline 1 & 0 & 0 & 0 & 0 & 0 & 1 & 0 & 1 \\
1 & 1 & 0 & 0 & 0 & 0 & 0 & 1 & 0 \\
0 & 1 & 1 & 0 & 0 & 0 & 0 & 0 & 1 \\
1 & 0 & 1 & 1 & 0 & 0 & 0 & 0 & 0 \\
0 & 1 & 0 & 1 & 1 & 0 & 0 & 0 & 0 \\
0 & 0 & 1 & 0 & 1 & 1 & 0 & 0 & 0 \\
0 & 0 & 0 & 1 & 0 & 1 & 1 & 0 & 0 \\
0 & 0 & 0 & 0 & 1 & 0 & 1 & 1 & 0 \\
\hline 0 & 0 & 0 & 0 & 0 & 0 & 1 & 1 & 1 \\
\hline 0 & 0 & 0 & 1 & 1 & 1 & 0 & 0 & 0 \\
1 & 1 & 1 & 0 & 0 & 0 & 0 & 0 & 0 \\
\hline 0 & 0 & 0 & 0 & 0 & 1 & 1 & 1 & 0 \\
\hline 1 & 1 & 0 & 0 & 0 & 0 & 0 & 0 & 1 \\
0 & 0 & 1 & 1 & 1 & 0 & 0 & 0 & 0 \\
\hline
\end{tabular}

Fig. 4. The solution of dimension $15 \times 9$ obtained by applying $\operatorname{Rec}(H, V, \mathcal{E})$ when the horizontal projections have constant value 3 , and the vertical projections 5

The validity of $\operatorname{Rec}(H, V, \mathcal{E})$ is a simple consequence of Theorem 3 . Clearly, the obtained matrix has homogeneous horizontal and vertical projections, equal to $h$ and $v$, respectively, and, by construction, all the rows are distinct. Moreover, the algorithm always terminates since at each iteration, we add as many rows as possible to the final solution. Concerning the complexity analysis, we need to generate $O(m)$ different Lyndon words and shift each of them $O(n)$ times. So, since the algorithm $\operatorname{GenLyndon}(n, h)$ requires $O(k \cdot h \cdot n)$ steps to generate $k$ words of $L(n, h)$, the whole process takes polynomial time.

We conclude the paper by observing that our strategy could be suitably extended to a larger class of binary matrices with different rows, homogeneous horizontal projections, where the vertical projections are "quasi-homogeneous", in the sense that their differences are bounded by a constant value.

\section{References}

1. Balogh, E., Sorantin, E., Nyúl, L.G., Palágyi, K., Kuba, A., Werkgartner, G., Spuller, E.: Virtual dissection of the colon: technique and first experiments with artificial and cadaveric phantoms. In: Medical Imaging 2002: Image Processing, San Diego, USA. Proceedings of SPIE, vol. 4681, pp. 713-721 (2002)

2. Batenburg, K.J., Bals, S., Sijbers, J., Kuebel, C., Midgley, P.A., Hernandez, J.C., Kaiser, U., Encina, E.R., Coronado, E.A., Van Tendeloo, G.: 3D imaging of nanomaterials by discrete tomography. Ultramicroscopy 109(6), 730-740 (2009)

3. Bentz, C., Costa, M.C., Picouleau, C., Ries, B., de Werra, D.: Degree-constrained edge partitioning in graphs arising from discrete tomography. Journal of Graph Algorithms and Applications 13(2), 99-118 (2009)

4. Berge, C.: Graphes. Gauthier-Villars, Paris (1983)

5. Berge, C.: Hypergraphs. North Holland (1989)

6. Brocchi, S., Frosini, A., Picouleau, C.: Reconstruction of binary matrices under fixed size neighborhood constraints. Theoretical Computer Science 406(1-2), 43$54(2008)$ 
7. Brocchi, S., Frosini, A., Rinaldi, S.: A reconstruction algorithm for a subclass of instances of the 2-color problem. Theoretical Computer Science 412, 4795-4804 (2011)

8. Brocchi, S., Frosini, A., Rinaldi, S.: The 1-Color Problem and the Brylawski Model. In: Brlek, S., Reutenauer, C., Provençal, X. (eds.) DGCI 2009. LNCS, vol. 5810, pp. 530-538. Springer, Heidelberg (2009)

9. Dürr, C., Guiñez, F., Matamala, M.: Reconstructing 3-colored grids from horizontal and vertical projections is NP-hard: A Solution to the 2-Atom Problem in Discrete Tomography. SIAM J. Discrete Math. 26(1), 330-352 (2012)

10. Gale, D.: A theorem on flows in networks. Pacific J. Math. 7, 1073-1082 (1957)

11. Gardner, R.J., Gritzmann, P.: Discrete tomography: determination of finite sets by X-rays. Trans. Amer. Math. Soc. 349, 2271-2295 (1997)

12. Gilbert, E.N., Riordan, J.: Symmetry types of periodic sequences. Illinois J. Math. 5, 657-665 (1961)

13. Guiũez, F., Matamala, M., Thomassé, S.: Realizing disjoint degree sequences of span at most two: A tractable discrete tomography problem. Discrete Applied Mathematics 159(1), 23-30 (2011)

14. Herman, G.T., Kuba, A.: Discrete tomography: Foundations algorithms and applications. Birkhauser, Boston (1999)

15. Herman, G.T., Kuba, A.: Advances in Discrete Tomography and Its Applications. Birkhauser, Boston (2007)

16. Irving, R.W., Jerrum, M.R.: Three-dimensional statistical data security problems. SIAM Journal of Computing 23, 170-184 (1994)

17. Matej, S., Vardi, A., Hermann, G.T., Vardi, E.: Binary tomography using Gibbs priors. In: Herman, G.T., Kuba, A. (eds.) Discrete Tomography: Foundations, Algorithms and Applications, pp. 191-212. Birkhauser, Boston (1999)

18. Prause, G.P.M., Onnasch, D.G.W.: Binary reconstruction of the heart chambers from biplane angiographic image sequence. IEEE Transactions on Medical Imaging 15, 532-559 (1996)

19. Ryser, H.: Combinatorial Mathematics. Mathematical Association of America and Quinn \& Boden, Rahway (1963)

20. Ryser, H.J.: Combinatorial properties of matrices of zeros and ones. Canadian Journal of Mathematics 9, 371-377 (1957)

21. Ruskey, F., Sawada, J.: An efficient algorithm for generating necklaces with fixed density. Siam J. Comput. 29, 671-684 (1999)

22. Sawada, J.: A fast algorithm to generate necklaces with fixed content. Theoret. Comput. Sci. 301, 477-489 (2003)

23. Shliferstein, A.R., Chien, Y.T.: Switching components and the ambiguity problem in the reconstruction of pictures from their projections. Pattern Recognition 10, 327-340 (1978) 